\title{
Obstrucción intestinal secundaria a hernia de Spiegel estrangulada con contenido de íleon terminal, apéndice cecal, ciego y colon ascendente. Reporte de un caso
}

\author{
Strangulated spigelian hernia with contents of the terminal ileum, \\ cecal appendix, cecum and ascending colon. Report of a case \\ María de Jesús Rupit Serrano,* Álvaro Rodrigo Jaime Limón, ** \\ Fernando Palacio Vélez***
}

Palabras clave: Hernia de Spiegel, hernia spigeliana, hernia de la línea semilunar, hernia intersticial, hernia estrangulada, obstrucción intestinal.

Key words: Spiegel hernia, spigelian hernia, semilunar line hernia, interstitial hernia, strangulated hernia, intestinal obstruction.

\section{RESUMEN}

Introducción: La hernia de Spiegel representa del 0.12 al $2 \%$ de las hernias de la pared abdominal; es un defecto en la aponeurosis de los músculos transverso y oblicuos del abdomen, entre el borde lateral de la vaina del recto abdominal y la línea semilunar. Presenta incarceración en $10.4 \%$ y estrangulación en $6.7 \%$. El contenido herniado usualmente es el intestino delgado y el epiplón. Caso clínico: Mujer de 80 años, con antecedente de ocho meses de aumento progresivo de volumen en el flanco abdominal derecho, con tres días de cuadro de obstrucción intestinal y dolor abdominal. Se realizó una tomografía de abdomen simple, en la que se encontró un anillo herniario de $39 \mathrm{~mm}$ adyacente a los músculos transverso y oblicuo menor del hemiabdomen derecho, lateral al músculo recto abdominal, con protrusión del colon ascendente, que le condicionaba obstrucción intestinal. Objetivo: Describir un caso clínico de hernia de Spiegel estrangulada, el cual se presentó como abdomen agudo secundario a obstrucción intestinal. Su dimensión y contenido fueron infrecuentes; este incluyó íleon terminal, ciego, apéndice cecal y colon ascendente. Conclusiones: La hernia de Spiegel es rara. El cirujano necesita el conocimiento de la patología para mantener un alto grado de sospecha en pacientes con factores de riesgo, realizar una amplia anamnesis y examen físico, apoyándose de estudios de imagen. El tratamiento definitivo es el quirúrgico.

\section{ABSTRACT}

Introduction: Spigelian hernia represents 0.12 to $2 \%$ of the abdominal wall hernias; it is a defect in the aponeurosis of the transverse and oblique muscles, between the lateral border of the sheath of the rectus abdominis muscle and the semilunar line. It presents incarceration in $10.4 \%$ and strangulation in $6.7 \%$. The herniated content is usually the small bowel and the omentum. Case report: An 80-yearold woman, with a history of eight months of progressive increase of volume in the right side flank, came to us with three days of intestinal obstruction and abdominal pain. A simple abdominal tomography was performed, finding an aponeurotic defect of $39 \mathrm{~mm}$ adjacent to the transverse and minor oblique muscles, with protrusion of the ascending colon that conditioned intestinal obstruction. Objective: To report a clinical case of Spiegel's strangulated hernia, which presented as an acute abdomen secondary to intestinal obstruction. Its size and content were uncommon, and included terminal ileum, cecum, cecal appendix and ascending colon. Conclusions: Spiegel's hernia is a rare defect; a challenge that usually leads to delayed diagnosis, which allows the development of clinical complications. The surgeon needs the knowledge of the pathology to keep a high degree of suspicion in patients with risk factors, in order to carry out a wide anamnesis and physical examination, relying on imaging studies. The definitive treatment is surgery.

\section{INTRODUCCIÓN}

$\mathrm{L}$ a reparación de los defectos herniarios de la $\checkmark$ pared abdominal es una de las intervenciones quirúrgicas más comunes en cirugía gene- ral, tanto de forma urgente como programada; ${ }^{1}$ representó 150,000 egresos hospitalarios en el año $2008 .^{2}$

El cirujano afrontará hernias que por su localización y contenido son consideradas 
inusuales, que dan lugar a cuadros clínicos que requieren un alto grado de sospecha y el uso de estudios de imagen para el diagnóstico prequirúrgico; tal es el caso de la hernia de Spiegel, también conocida como hernia de la línea semilunar o ventrolateral.

La hernia de Spiegel es una entidad infrecuente: no supera una prevalencia del $2 \%$ dentro de todas las hernias de la pared abdominal. ${ }^{3}$ El contenido más común es el intestino delgado y el epiplón. ${ }^{4}$

Al momento de su presentación, alrededor de $90 \%$ son asintomáticas; ${ }^{5}$ hasta $50 \%$ de los casos son diagnosticados incorrectamente ${ }^{6}$ y la tasa de complicaciones es alta $(21.1 \%){ }^{7}$

El objetivo de este reporte es describir un caso clínico de hernia de Spiegel estrangulada, la cual se presentó como abdomen agudo secundario a obstrucción intestinal. Su contenido era infrecuente e incluyó íleon terminal, ciego, apéndice cecal y colon ascendente.

\section{CASO CLÍNICO}

Se trató de mujer de 80 años de edad, con antecedente de hipertensión arterial sistémica de 10 años de diagnóstico, en tratamiento médico con captopril 25 mg vía oral cada 24 horas; cáncer de mama izquierda diagnosticado tres años atrás, manejado con mastectomía radical; actualmente, en quimioterapia con anastrozol 1 mg vía oral cada 24 horas. Se sometió a plastía umbilical 20 años atrás, sin complicaciones aparentes. Negó antecedentes traumáticos, transfusionales, alérgicos, etcétera.

El padecimiento actual inició tres días previos a su ingreso al Servicio de Urgencias, con presencia de dolor abdominal de tipo opresivo y cólico en el hemiabdomen derecho, estreñimiento, ataque al estado general y emesis de características fecaloides en cinco ocasiones; por este motivo fue llevada por familiares al Servicio de Urgencias. Al interrogatorio dirigido, refirió un aumento de volumen progresivo en el flanco derecho de ocho meses de evolución, sin desencadenante aparente, sin valoración médica previa.

A la exploración física de ingreso, se encontró con signos vitales con tendencia a la hipotensión, afebril, somatotipo endomórfico, índice de masa corporal de $39.7 \mathrm{~kg} / \mathrm{m}^{2}$. Neu- rológicamente: consciente, desorientada en las tres esferas, poco cooperadora, con palidez generalizada de tegumentos, mal estado de hidratación en las mucosas, cuello cilíndrico, campos pulmonares con disminución del murmullo vesicular en ambas bases, ruidos cardiacos de adecuada frecuencia y disminuidos en intensidad. Abdomen globoso a expensas de panículo adiposo y distensión abdominal, peristalsis ausente; el flanco derecho, con aumento de volumen, sin cambios de coloración. A la palpación, se identificó la presencia de un tumor de consistencia blanda, móvil, profunda, de bordes no definidos, no reductible, con aumento de volumen con la maniobra de Valsalva. El resto del abdomen, doloroso a la palpación generalizada, con descompresión positiva. Extremidades íntegras, eumétricas, sin compromiso neurovascular aparente. A la colocación de la sonda nasogástrica, con gasto fecaloide.

La radiografía portátil simple de abdomen en posición de decúbito dejó ver dilatación de asas lateralmente, mostrando los pliegues de las haustras, ausencia de gas y materia fecal en recto sigma (Figura 1).

Los laboratorios a su ingreso: leucocitos de $4 \times 10^{6} / \mathrm{mm}$, hemoglobina $12.7 \mathrm{~g} / \mathrm{dl}$, hematocrito $37.5 \%$, plaquetas $169 \times 10^{6} / \mathrm{mm}^{3}$, glucosa

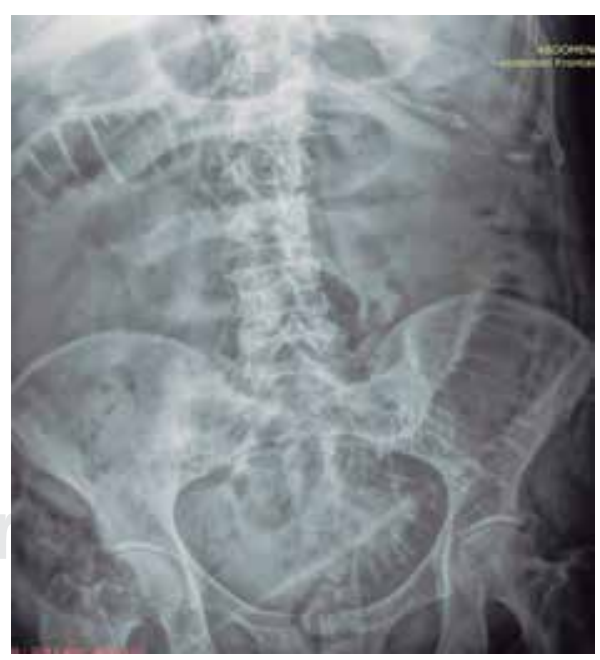

Figura 1. Radiografía portátil simple de abdomen en posición de decúbito con dilatación de asas del intestino delgado; muestra válvulas conniventes. No se observa gas distal. 
$291 \mathrm{mg} / \mathrm{dl}$, urea $57 \mathrm{mg} / \mathrm{dl}$, nitrógeno ureico 27 $\mathrm{mg} / \mathrm{dl}$, creatinina $0.7 \mathrm{mg} / \mathrm{dl}$, sodio $129 \mathrm{mmol} / \mathrm{l}$, potasio $4.1 \mathrm{mmol} / \mathrm{l}$, cloro $103 \mathrm{mmol} / \mathrm{l}$, calcio $5.7 \mathrm{mmol} / \mathrm{l}$, INR 1.4 .

La tomografía simple de abdomen realizada 24 horas posteriores a su ingreso al Servicio de Urgencias mostró defecto aponeurótico de 39 $\mathrm{mm}$ adyacente al borde lateral del músculo recto abdominal derecho y a los músculos transverso y oblicuo menor derecho, por donde protruían asas de intestino delgado, ciego y colon ascendente, con dilatación de asas del intestino delgado proximales al sitio de obstrucción (Figura 2). Como hallazgos adicionales, se identificó la presencia de escaso derrame pleural y pericárdico.

Se programó para cirugía de urgencia, previo consentimiento informado, para laparotomía exploradora y plastía de pared, de la cual se realizó una incisión oblicua de 10 $\mathrm{cm}$ sobre el defecto herniario; se profundizó por planos hasta exponer la aponeurosis del músculo oblicuo mayor (Figura 3). Se incidió en el sentido de sus fibras, teniendo como hallazgo un saco herniario de aproximadamente $10 \times 5 \mathrm{~cm}$ interparietal. Debajo del nivel umbilical paramedial derecho (Figura 4), se realizó disección roma y apertura del saco herniario; se encontró escaso líquido turbio y fétido, íleon terminal, válvula ileo-

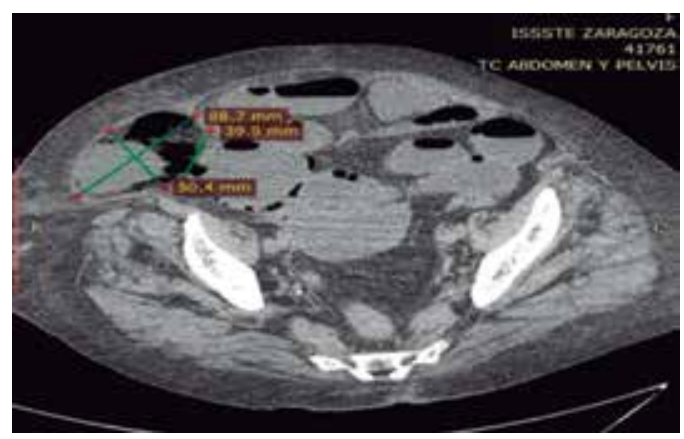

Figura 2. Tomografía simple de abdomen en corte axial; muestra debajo de la aponeurosis del músculo oblicuo externo derecho un saco con dimensión máxima de 88.7 $\mathrm{mm}$ de largo, $50.4 \mathrm{~mm}$ de ancho, con anillo herniario de $39.9 \mathrm{~mm}$ a nivel de los planos musculares, por donde protruyen asas del intestino delgado, con presencia de nivel hidroaéreo, con dilatación de asas del intestino delgado previas al sitio de obstrucción. cecal, ciego, segmento proximal del colon ascendente necrosados y epiplón con zonas isquémicas (Figura 5).

Se realizó en forma simultánea laparotomía exploradora, en la que se halló distensión de asas del intestino delgado proximales al sitio de estrangulación, peritoneo parietal periférico al anillo herniario de coloración necrótica.

Se redujo el contenido herniado hacia la cavidad abdominal, lo que reveló un orificio herniario de aproximadamente cuatro centímetros de diámetro.

Se realizó hemicolectomía derecha con cierre distal del colon en bolsa de Hartmann con sutura manual, en dos planos de sutura, poliglactina 910 3-0 para el plano mucoso y puntos de Lembert con seda 3-0 para el plano seromuscular, más ileostomía terminal de Brooke, lavado de cavidad abdominal con

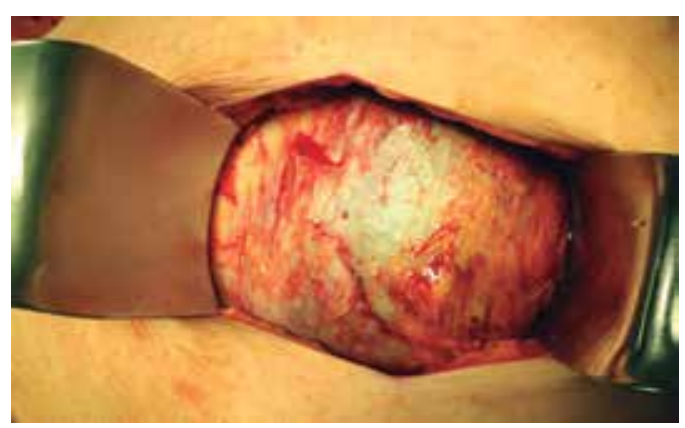

Figura 3. Apertura de piel y tejido celular subcutáneo, con exposición de la aponeurosis del músculo oblicuo externo, que se encuentra abombada por un saco herniario subyacente.

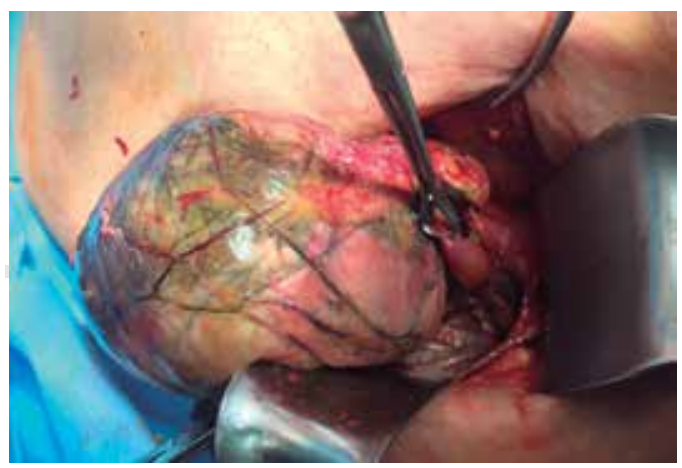

Figura 4. Saco herniario disecado e íntegro, con presencia de anillo herniario en su base. 


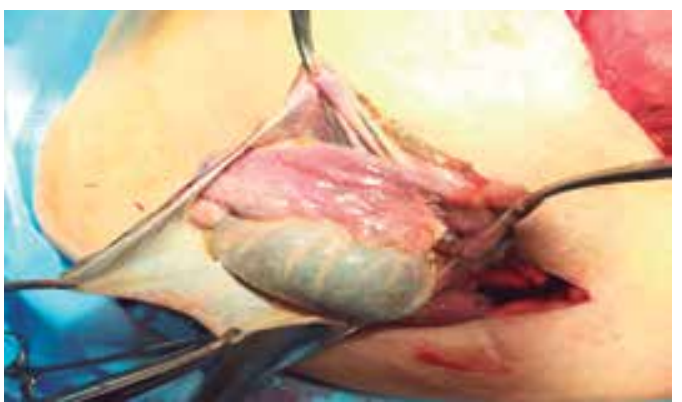

Figura 5. Apertura del saco herniario, con epiplón en la parte superior y asa de intestino delgado dilatada en la parte inferior.

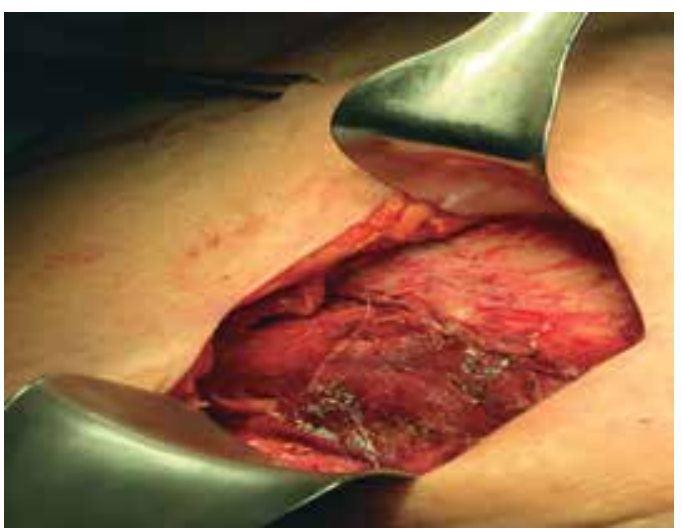

Figura 6. Cierre anatómico de la aponeurosis del músculo oblicuo derecho.

colocación de drenaje tipo Penrose de media pulgada abocado al hueco pélvico.

Por tratarse de una herida contaminada, se decidió la reparación anatómica por planos, cerrando la aponeurosis de los músculos transverso y oblicuos del abdomen con sutura continua de polidioxanona 1-0 (Figura 6), colocación de drenaje Penrose de 5/16 de pulgada a nivel del tejido celular subcutáneo, cierre de piel con nylon del 2-0, con puntos de Sarnoff. El cierre de la laparotomía exploradora fue con catgut crómico de 1-0 para peritoneo parietal, aponeurosis con tripletas de polidioxanona 1-0; se afrontó el tejido celular subcutáneo con puntos simples de catgut crómico 2-0, piel con nylon 2-0, con puntos de Sarnoff.

Desarrolló neumonía nosocomial al séptimo día de operada, a pesar del manejo antibiótico intravenoso instaurado en el

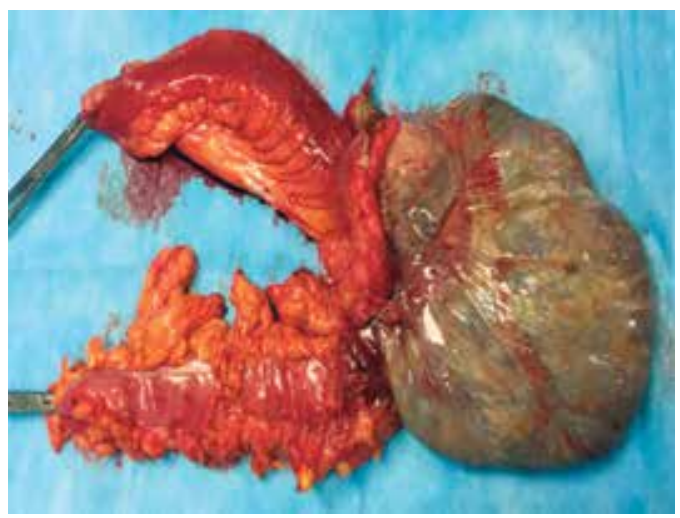

Figura 7. Imagen del segmento resecado; el asa superior corresponde al íleon terminal, el asa inferior es el segmento del colon ascendente resecado. Se observa a la derecha el saco herniario y su contenido, con cambios de coloración.

postquirúrgico inmediato con levofloxacino 500 mg cada 24 horas y metronidazol 500 mg cada ocho horas por 14 días, por lo que permaneció 20 días en estancia intrahospitalaria. Egresó a su domicilio por mejoría. Se le dio seguimiento por seis meses en la consulta externa del Servicio de Cirugía General, sin complicaciones ni recurrencia.

El informe anatomopatológico de la pieza enviada (Figura 7) concluyó: peritonitis aguda y crónica no específica, necrosis de la pared a nivel del colon ascendente, ciego, apéndice cecal e íleon terminal; tejido fibroconectivo (saco herniario) con inflamación crónica.

\section{DISCUSIÓN}

Adriaan van den Spiegel (1578-1625), anatomista belga, describió por primera vez la línea semilunar que ahora lleva su nombre, que se define como la línea de transición entre los haces musculares y la aponeurosis del músculo transverso del abdomen, así como su relación con el borde lateral del músculo recto abdominal (fascia de Spiegel). ${ }^{8}$ Fue hasta 1764 que Joseph Thaddaeus Klinkosch, cirujano anatomista de Praga, describió por primera vez este defecto. ${ }^{9}$ El 90\% de estas hernias se ubica en el cinturón de Spangen, que corresponde a una franja transversal de seis centímetros ubicada entre la altura de la cicatriz umbilical 
y la línea interespinosa. ${ }^{10}$ La hernia de Spiegel tiene su punto de debilidad en la aponeurosis del músculo transverso del abdomen, entre el borde lateral de la vaina del recto abdominal y la línea semilunar. La mayoría penetra los músculos transversos del abdomen y oblicuo interno, y se sitúa debajo del músculo oblicuo mayor; son hernias intersticiales y ocultas. ${ }^{11}$ En la mayor parte de los casos, el tamaño del anillo herniario es de 0.5 a dos centímetros de diámetro. ${ }^{12}$ El concepto aún no estandarizado de hernia gigante de Spiegel ha sido descrito para nombrar diversos tamaños, de entre 4 y 10 centímetros para el anillo herniario y de entre 10 y 18 centímetros del saco herniario. ${ }^{4}$

Presenta una prevalencia del 0.12 al $2 \%$ dentro de todas las hernias de pared abdominal, ${ }^{13}$ con predominio a partir de la quinta década de la vida. ${ }^{14}$ La mayoría de los defectos son adquiridos y no congénitos. ${ }^{15}$ Ocurre con más frecuencia en mujeres, con una relación mujer:hombre de 1.4:1.16 La proporción entre las del lado derecho y el izquierdo es similar, pero algunas series informan predominio del lado izquierdo; ${ }^{17}$ excepcionalmente, existen bilaterales.

Del 62.5 al $79 \%$ presentan factores de riesgo como la obesidad, enfermedad pulmonar obstructiva crónica, cirugías previas (incluyendo laparoscópicas), traumatismos abdominales, multiparidad, estreñimiento y ascitis. ${ }^{18}$

El epiplón mayor y las asas intestinales son el contenido herniado más frecuente; de forma excepcional, se han descrito apéndice, colon, estómago y ovario. ${ }^{19}$

Al momento de su presentación, del 80 al $90 \%$ son asintomáticas y se encuentran como hallazgo incidental durante exploraciones físicas o procedimientos quirúrgicos adicionales. ${ }^{4}$

La hernia de Spiegel no tiene una sintomatología característica. El dolor abdominal ocurre en el 31-86\%; este es sordo, de localización imprecisa y larga evolución, aumenta con la maniobra de Valsalva y se atenúa con el decúbito. ${ }^{20}$ En $50 \%$ hay sensación de masa abdominal, que se reduce con el decúbito. Otros síntomas son náusea, vómito, hiperestesia local y alteración del ritmo intestinal. ${ }^{21}$

A la exploración física, en 35\% puede palparse una masa blanda y profunda, ${ }^{22}$ con peristalsis en caso de contener asas intestinales, excepto en las hernias pequeñas, de localización interparietal o en pacientes con obesidad. ${ }^{23}$

El retraso diagnóstico es habitual y hasta $50 \%$ de los casos son diagnosticados incorrectamente. ${ }^{5}$ Por lo anterior, la tasa de complicaciones es alta: $35.5 \%$; incarceración 10.4\%, estrangulación $6.7 \%$, obstrucción intestinal $12.9 \%$ y suboclusión $5.5 \%{ }^{24}$

El diagnóstico diferencial se realiza con procesos intraabdominales y parietales. Respecto de los primeros, se pueden citar ejemplos como diverticulitis, tumores de colon, implante tumoral peritoneal, tumoración pélvica, embarazo ectópico, quiste de ovario, obstrucción intestinal, apendicitis, trastornos genitourinarios. En cuanto a los procesos parietales: hematoma de los músculos rectos, miotendinitis, sarcoma, tumor desmoide, lipoma y hernias inguinales de trayecto ascendente. ${ }^{25}$

En los casos de duda diagnóstica, está indicado el uso de estudios de imagen. La ecografía es la exploración más útil, mientras que la tomografía aporta el diagnóstico definitivo, localización y tamaño exacto del defecto, las relaciones de vecindad y contenido del saco, necesarios para determinar el abordaje quirúrgico. ${ }^{26}$

Debido al alto riesgo de complicaciones, la única modalidad terapéutica es la quirúrgica, ya sea de forma urgente o programada; con disección, apertura y exéresis del saco herniario, tratamiento de su contenido, cierre del anillo herniario y reforzamiento con las estructuras aponeuróticas vecinas. Se recomienda la reparación con malla en anillos herniarios mayores a dos centímetros. Desde 1992 se introdujo su manejo laparoscópico, vía preperitoneal o intraabdominal; ha mostrado ventaja al reducir la morbilidad y reducir la incisión y disección necesarias para la exposición del defecto. ${ }^{27}$

El riesgo de recurrencia es de $2 \%$, similar al reportado para otras hernias de la pared abdominal. ${ }^{28}$

El caso particular de esta paciente refleja concordancia con la literatura respecto a factores epidemiológicos, como su género, edad, obesidad y tiempo evolutivo; así como hallazgos clínicos que establecieron la sospecha diagnóstica de hernia de Spiegel, que se confirmó por tomografía. Sin embargo, este caso clínico fue excepcional por el tamaño del anillo 
y el saco herniario, así como por el contenido herniado. Sólo se han encontrado siete casos de obstrucción intestinal por compromiso del colon en la revisión de la literatura. ${ }^{29}$

\section{CONCLUSIÓN}

La obstrucción intestinal por hernia de Spiegel estrangulada es infrecuente. Estas hernias son un reto por hallarse intersticiales y con clínica no característica, lo que usualmente produce un retraso en el diagnóstico y permite el desarrollo de complicaciones clínicas. El cirujano necesita el conocimiento de la patología para mantener un alto grado de sospecha en pacientes con factores de riesgo, realizar una amplia anamnesis y examen físico, apoyándose de estudios de imagen. La tomografía computarizada muestra la anatomía y el contenido del saco herniario, lo que permite la planificación quirúrgica. Dada la alta tasa de encarcelamiento y estrangulación, el tratamiento definitivo es el quirúrgico; la reparación abierta es segura en escenarios de urgencia para hernias complicadas y se acompaña de excelentes resultados.

\section{Agradecimientos}

Al Servicio de Cirugía General y Servicio de Radiología e Imagen del Hospital Regional "General Ignacio Zaragoza", Instituto de Seguridad y Servicios Sociales de los Trabajadores del Estado.

\section{REFERENCIAS}

1. Martínez-Serrano MA, Pereira JA, Sancho JJ, LópezCano M, Bombuy E, Hidalgo J, et al. Risk of death after emergency repair of abdominal wall hernias. Still waiting for improvement. Langenbecks Arch Surg. 2010; 395: 551-556.

2. Guías de Práctica Clínica para Hernias de la Pared Abdominal. Asociación Mexicana de Hernia, AC. México, agosto de 2015: 5-7.

3. Montes IS, Deysine M. Spigelian and other uncommon hernia repairs. Surg Clin North Am. 2003; 83: 12351253, viii.

4. Skandalakis PN, Zoras O, Skandalakis JE, Mirilas P. Spigelian hernia: surgical anatomy, embryology, and technique of repair. Am Surg. 2006; 72: 42-48.

5. Gil-Galindo G, Romero-González RJ, Flores-Salinas MA, Romero-González R, Pulido-Rodríguez J. Hernia gigante de Spiegel y traumatismo abdominal: ¿causa o coincidencia? Cir Cir. 2012; 80: 189-192.
6. Stirnemann H. The Spigelian hernia: missed? rare? puzzling diagnosis? Chirurg. 1982; 53: 314-317.

7. Artioukh DY, Walker SJ. Spigelian herniae: presentation, diagnosis and treatment. J R Coll Surg Edinb. 1996; 41: 241-243.

8. Moreno-Egea A. Controversias históricas de las hernias semilunares: Spiegel frente a Casseri, Klinkosch frente a Molliére. Rev Hispanoam Hernia. 2014; 2: 119-125.

9. Vos DI, Scheltinga MR. Incidence and outcome of surgical repair of spigelian hernia. Br J Surg. 2004; 91 : 640-644.

10. Spangen L. Spigelian hernia. World J Surg. 1989; 13: 573-580.

11. Martínez-García M, González-Uriarte J, Irazusta M, Gurruchaga JM, Álvarez-Caperochipi J, Mendoza M, et al. Hernia de Spiegel. Diagnóstico y epidemiología. Cir Esp. 2000; 68: 74-76.

12. Salemis NS, Kontoravdis N, Gourgiotis S, Panagiotopoulos N, Gakis C, Dimitrakopoulos G. Colonic obstruction secondary to incarcerated Spigelian hernia in a severely obese patient. Int J Surg Case Rep. 2010; 1: 27-29.

13. Houlihan TJ. A review of Spigelian hernias. Am J Surg. 1976; 131: 734-735.

14. Adell-Carceler R, Salvador-Sanchís JL, Laguna-Sastre M, Navarro-Navarro J, Gibert-Gerez J, Escrig-Sos J, et al. Hernia de Spiegel. Formas de presentación clínica y alternativas terapéuticas. Cir Esp. 1996; 60: 14-16.

15. Durham MM, Ricketts RR. Congenital spigelian hernias and cryptorchidism. J Pediatr Surg. 2006; 41: 18141817.

16. Read RC. Observations on the etiology of spigelian hernia. Ann Surg. 1960; 152: 1004-1009.

17. Moreno-Egea A, Carrasco L, Girela E, Martín JG, Aguayo JL, Canteras M. Open vs laparoscopic repair of spigelian hernia: a prospective randomized trial. Arch Surg. 2002; 137: 1266-1268.

18. Cerdán-Pascual R, Cantín-Blázquez S, BarrancoDomínguez JI, Bernal-Jaulín J, Duque-Mallén MV. Hernia de Spiegel: revisión a propósito de 8 casos. Rev Cubana Cir. 2005; 44 (4). Disponible en: http://scielo. sld.cu/scielo.php?script $=$ sci_arttext \&pid $=$ S0034$74932005000400007 \& \operatorname{lng}=\mathrm{es}^{-}$.

19. Ramírez-Ramírez MM, Villanueva-Sáenz E. Hernias infrecuentes con contenido atípico. A propósito de una hernia de Spiegel con apendicitis aguda. Rev Gastroenterol Mex. 2017; 82: 181-182.

20. Artioukh DY, Walker SJ. Spigelian herniae: presentation, diagnosis and treatment. J R Coll Surg Edinb. 1996; 41: 241-243.

21. Malazgirt Z, Topgul K, Sokmen S, Ersin S, Turkcapar AG, Gok H, et al. Spigelian hernias: a prospective analysis of baseline parameters and surgical outcome of 34 consecutive patients. Hernia. 2006; 10: 326330.

22. Larson DW, Farley DR. Spigelian hernias: repair and outcome for 81 patients. World J Surg. 2002; 26: 1277-1281.

23. Zaragoza C, Villalba S, Castaño S, Trullenque R. Hernia de Spiegel. Cir Esp. 1996; 59: 165-167.

24. Moles-Morenilla L, Docobo-Durántez F, Mena-Robles J, de Quinta-Frutos R. Spigelian hernia in Spain: an 
analysis of 162 cases. Rev Esp Enferm Dig. 2005; 97: 338-347.

25. Cruz-Rodríguez J, Cinta-Domínguez L, Pérez-Cedeño Y. Hernia de Spiegel. A propósito de un caso. Rev Haban Cienc Méd. 2014; 13: 751-756.

26. Martin M, Paquette B, Badet N, Sheppard F, Aubry S, Delabrousse E. Spigelian hernia: CT findings and clinical relevance. Abdom Imaging. 2013; 38: 260-264.

27. Richards AT. Spigelian hernias. Oper Tech Gen Surg. 2004; 6: 228-239.

28. Mathonnet $M$, Mehinto D. Anterolateral hernias of the abdomen. J Chir (Paris). 2007; 144 Spec No 4: 5S19-5S22.

29. Salemis NS, Kontoravdis N, Gourgiotis S, Panagiotopoulos N, Gakis C, Dimitrakopoulos G. Colonic obstruction secondary to incarcerated
Spigelian hernia in a severely obese patient. Int J Surg Case Rep. 2010; 1: 27-29.

Correspondencia:

\section{María de Jesús Rupit Serrano}

Servicio de Cirugía General, Hospital Regional "General Ignacio Zaragoza",

Instituto de Seguridad y Servicios Sociales de los Trabajadores del Estado.

Calz. Gral. Ignacio Zaragoza Núm. 1711, Col. Ejército Constitucionalista, 09220,

Iztapalapa, Ciudad de México, México. Tel.: +525557165200

E-mail: rupit.jaime@gmail.com 